\title{
Decomposed but Parallel Processing of Two-Digit Numbers in 1st Graders
}

\author{
S. Pixner ${ }^{1,2, \#}$, K. Moeller, ${ }^{2,3, \#, *}$, J. Zuber ${ }^{2,3}$ and H.-C. Nuerk ${ }^{2,3}$ \\ ${ }^{I}$ Department for Child and Adolescent Psychiatry, University Ulm, Ulm, Germany \\ ${ }^{2}$ Department of Psychology, Paris-Lodron University Salzburg, Salzburg, Austria \\ ${ }^{3}$ Department of Psychology, Eberhard Karls University, Tuebingen, Germany
}

\begin{abstract}
It has been suggested that decomposed processing of two-digit numbers develops from sequential (left-to-right) to parallel with age (Nuerk et al., 2004). However, task demands may have provoked sequential processing as a specific rather than a universal processing style. In the current study a standard unit-decade compatibility effect observed in two-digit number magnitude comparison indicated that first graders were already able to process the single digit magnitudes of tens and units separately and in parallel. Consequently, previous findings of sequential processing may be specific for stimulus characteristics in which such a processing style is useful. It is concluded that even first graders seem to be able to adapt their individual processing styles depending on stimulus properties. More generally, this suggests that the manner by which children process two-digit numbers is strategically adaptive rather than fixed at a particular developmental stage.
\end{abstract}

Key Words: Children's number processing, magnitude comparison, compatibility effect.

\section{INTRODUCTION}

\section{Number Processing in Children}

In recent years increasing research interest was devoted to the development of early number processing abilities in children. While only relatively few studies were conducted to examine the development of multi-digit number processing in children, the early development of single-digit number processing abilities received much more interest.

Regarding single-digit processing, for instance, Siegler and Robinson [1] found that children not only manage to differentiate between different quantities, but that they are also able to compare Arabic numbers by magnitude as early as at the age of 4 years; even before these were learned at school. Moreover, Rubinsten, Henik, Berger and ShaharShalev [2] observed that already in first graders the magnitude of a presented Arabic digit is automatically activated even when it is irrelevant for the task at hand [see also 3,4]. However, not only the magnitude of the digit itself is activated, but also the (automatic) spatial representation of magnitude on a mental number line. From the finding that comparably smaller numbers (e.g., 1) were responded to faster with the left hand whereas for relatively larger numbers (e.g., 8) response latencies of the right hand were shorter, Dehaene, Bossini, and Gireaux [5] inferred that this

*Address correspondence to this author at the Eberhard Karls University Tuebingen, Department of Psychology, Friedrichstrase 21, 72072 Tuebingen, Germany; E-mail: korbinian.moeller@uni-tuebingen.de

"Both authors contributed equally to this manuscript and should be considered as shared first authors mental number line is oriented from left to right with increasing number magnitude being represented towards the right. In this context, van Galen and Reitsma [6, see also 7] observed that even in elementary school children (first grade) the mental number line is oriented in this way. So, to conclude, it is now fairly established that for single-digit numbers the magnitude of a number and its spatial representation on the mental number line are activated automatically in young children.

However, for multi-digit numbers little is known about how they are processed during early stages of numerical development: Are tens and units of two-digit numbers processed in a decomposed fashion (e.g., "24" as "2" and "4")? Or are two-digit numbers processed as one holistic entity, such as "24"? However, before considering the few developmental studies addressing this issue, evidence from adult two-digit number magnitude processing which is relevant for the current study will be reviewed briefly.

\section{Two-Digit Number Processing in Adults: Holistic or Decomposed?}

In recent years, there has been a vivid debate in the adult literature whether two-digit numbers are processed holistically or in a decomposed manner [5, 8-11, see 12 for a review]. In this debate, the holistic account suggests that the magnitudes of all two-digit numbers are represented on one single analogue (logarithmically compressed) mental number line with no specific reference to the base-10 structure of the Arabic number system (e.g., [13]). In contrast, the decomposed account suggests that the magnitudes of tens and units of two-digit numbers are (also) represented 
separately, possibly on concomitant number lines for tens and units, thereby complying with the base-10 structure of the Arabic number system (e.g., [14]). Yet, what is the evidence for these different accounts?

Dehaene, Dupoux, and Mehler [15] conducted a two-digit number comparison task in which they asked their participants whether a presented number (e.g., 47) was smaller or larger than a fixed standard (e.g., 65). They observed a logarithmic distance effect: The farther the number was away from the standard, the faster was the reaction time (RT, cf. [16]) and this distance effect followed a logarithmic function. Most importantly, for the current study were the results of the multiple regression analyses. Dehaene and colleagues [15] found that logarithmic overall distance was a sufficient predictor to account for the distance effect in their RT data. When this predictor was incorporated into the regression analysis any influence of the unit digits was no longer significant. These findings seemed to suggest that only the holistic (logarithmic) distance between two numbers is relevant for number comparison whereas there is no specific influence of the units on processing speed and accuracy. Therefore, these authors concluded that two-digit numbers are processed holistically as one analogue integrated entity.

However, the notion of number magnitude to be represented as one holistic entity was questioned in recent years. Nuerk, Weger and Willmes [10, 17, 18] observed a reliable influence of units in two-digit number magnitude comparison even when controlling for overall (holistic) distance. The important effect for the decomposed processing account is the unit-decade compatibility effect. Comparing two twodigit numbers can be differentiated in compatible and incompatible comparisons. When separate comparisons of tens and units would result in the same decision (e.g., for 42_57, both the decade and the unit digit of the larger number are larger than the corresponding digits of the smaller number, i.e., $4<5$ and $2<7$ ), the number pair is unit-decade compatible. In contrast, when separate comparisons of tens and units would result in different decisions (e.g., for 47_62, the decade digit of the larger number is also larger, but the unit digit of the larger number is smaller, i.e., $4<6$ but $7>2$ ), the number pair is unit-decade incompatible. Nuerk and colleagues repeatedly $[9,10,17,18]$ observed that the comparison of compatible number pairs (e.g., 42_57) was faster and less error prone than the comparison of incompatible pairs (e.g., 47_62). It is of major importance that overall (holistic) distance was matched between compatible and incompatible trials (e.g., 15 in both above examples). Therefore, a holistic account cannot explain the compatibility effect. Rather, the unit-decade compatibility effect indicates that two-digit numbers are (also) compared in a decomposed fashion and not only holistically [see also 19 - 23; and 12, for a review].

Thereby, the question arises how these different lines of evidence for holistic as well as for decomposed processing can be accounted for. Nuerk and Willmes [12, see also 9] have outlined in great detail why Dehaene and colleagues [15] and others may have failed to observe unit-based effects in their experimental design. We will not reiterate the whole line of reasoning, but outline the two most important arguments briefly.
First, the influence of unit distance has to be considered. Unit distance reflects the difference between the unit digits of the to-be-compared numbers (e.g., 31_58, 8 - 1 = 7). Nuerk and colleagues showed repeatedly [9, 10, 17] that the compatibility effect is more pronounced for larger unit distances (i.e., $\geq 4$ ). However, using a fixed standard ending on 5 (i.e., 55 or 65 ) limits the possible unit distance to a maximum of 4 (e.g., 55_41, $5-1=4$; 55_69; $9-5=4$ ). To overcome this, Nuerk and colleagues [10] introduced variable standards. This means that participants had to single out the larger of a pair of two-digit numbers instead of classifying a given number as smaller or larger than a fixed standard as in the former case unit distance varies between 1 and 8 (e.g., 36_47 and 31_59, respectively).

Second, the ratio of between- and within-decade trials in the stimuli set used is important. When no or only a small percentage of within-decade trials is included in the stimulus set $(15.5 \%$ in Dehaene et al. [15]) this seems to encourage participants to focus their decision on comparing the decade digits as these are decisive for the majority of trials. Such an attentional bias towards the decade digits in turn diminishes the influence of the unit digits. Consider now the same task when within-decade trials are included (e.g., 63_68). In such trials, the unit digits (i.e., 3 and 8 ) are decisive to determine the larger number as the decade digits are identical $[9 ; 22$; see 12 for a review]. Hence, by incorporating a considerable number of within-decade trials a processing style exclusively focusing on the decade digits is no longer beneficial as it implies focusing on the irrelevant digit in an important number of trials. These two factors have recently been demonstrated to be essential for parallel decomposed processing in adults [9]. Let us now elaborate how this has been considered in children studies.

\section{Two-Digit Number Processing in Children: Holistic or Decomposed?}

As for adults, children's reliance upon a holistic processing style would be reflected by the presence of a strong distance effect, but the absence of a unit-decade compatibility effect, as overall distance was held constant between compatible and incompatible trials. However, the results of Nuerk et al. [24] showed a reliable unit-decade compatibility effects suggesting decomposed processing of tens and units.

Yet, the mode of decomposed processing could be either parallel or sequential. This is indicated by a positive (i.e., compatible trials are processed faster than incompatible ones) or negative compatibility effect (i.e., incompatible trials are processed faster than compatible trials). A negative, respectively reversed, compatibility effect would indicate sequential left-to-right processing of tens and units as incompatible trials have a larger decade distance when controlling for overall distance: overall distance $=10 \times$ decade distance $+1 \times$ unit distance. Consider the above examples 42_57 vs. 47_62. For 42_57 overall distance is 15 $=10 \times(5-4$; i.e., decade distance $)+1 \times(7-2$; unit distance), so $10+5=15$. However, for 47_62 overall distance is $10 \times(6-4)+1 \times(2-7)$, so $20-5=15$. Thus, when overall distance is matched, decade distance must always be larger in incompatible trials because in these trials unit distance must be subtracted from rather than added to the decade distance [for a more detailed mathematical 
elaboration see 23,24$]$. Consequently, when processing the decade and unit digits sequentially from left to right, the decade digits are processed first and predominantly. This processing style should lead to shorter response latencies for incompatible number pairs as decade distance is larger for these number pairs. These shorter latencies in incompatible trials would then reflect the above-mentioned negative or reversed compatibility effect. Contrarily, parallel processing of tens and units would be indexed by a standard positive compatibility effect as observed for adults: Parallel decomposed processing of tens and units in incompatible number pairs would yield incongruent response biases causing interference which prolongs reaction times. In summary, parallel processing is indicated by a regular compatibility effect while sequential processing is associated with a negative compatibility effect [24].

This question whether two-digit numbers are processed in (i) holistic or in (ii) parallel decomposed or in (iii) sequential decomposed manner was studied in a developmental study by Nuerk and colleagues [24] in which they assessed children from $2^{\text {nd }}$ to $5^{\text {th }}$ grade. The authors found that children processed the magnitudes of tens and units in a decomposed fashion at all ages. However, the youngest children (i.e., second graders) processed tens and units separately, but in a sequential left-to-right fashion, meaning that these children start with comparing the decade digits. On the other hand, fifth graders seemed to rely entirely on separate but parallel processing of tens and units in a similar way as adults do. In between, there seemed to be a rather continuous transition from sequential (decomposed) to parallel (decomposed) processing with increasing age and experience (see Fig. 1).

To summarize, the results of Nuerk and colleagues [24] suggested two important hypotheses for the development of two-digit number processing. (i) They favoured the hypothesis that children process the magnitudes of tens and units separately rather than holistically. (ii) The results suggested that decomposed processing develops from initially sequential to more parallel processing with increasing age. Following this linear trend as observed by Nuerk et al. ([24] see Fig. 1), we would expect the reversal of the compatibility effect to be even more pronounced for $1^{\text {st }}$ graders. Please note that the children assessed by Nuerk et al. [24] as well as those recruited for the current study were tested in Austria. Thus, the present results should not be influenced by language and/or education/schooling differences.

\section{Processing Style Variation in Two-Digit Number Comparison Based on Stimulus Set}

As described in the section on adult two-digit number processing the configuration of the stimulus set exhibits a reliable influence on the results observed [see above and 9, $12,22]$. In adults, it was observed that the presence of a substantial proportion of within-decade trials - and consequently a more balanced relevance of decade and unit digits across the experiment - changes the processing style: The influence of unit digits becomes larger even in betweendecade trials when the unit digits are also relevant in a considerable number of trials [22]. When such effects can be

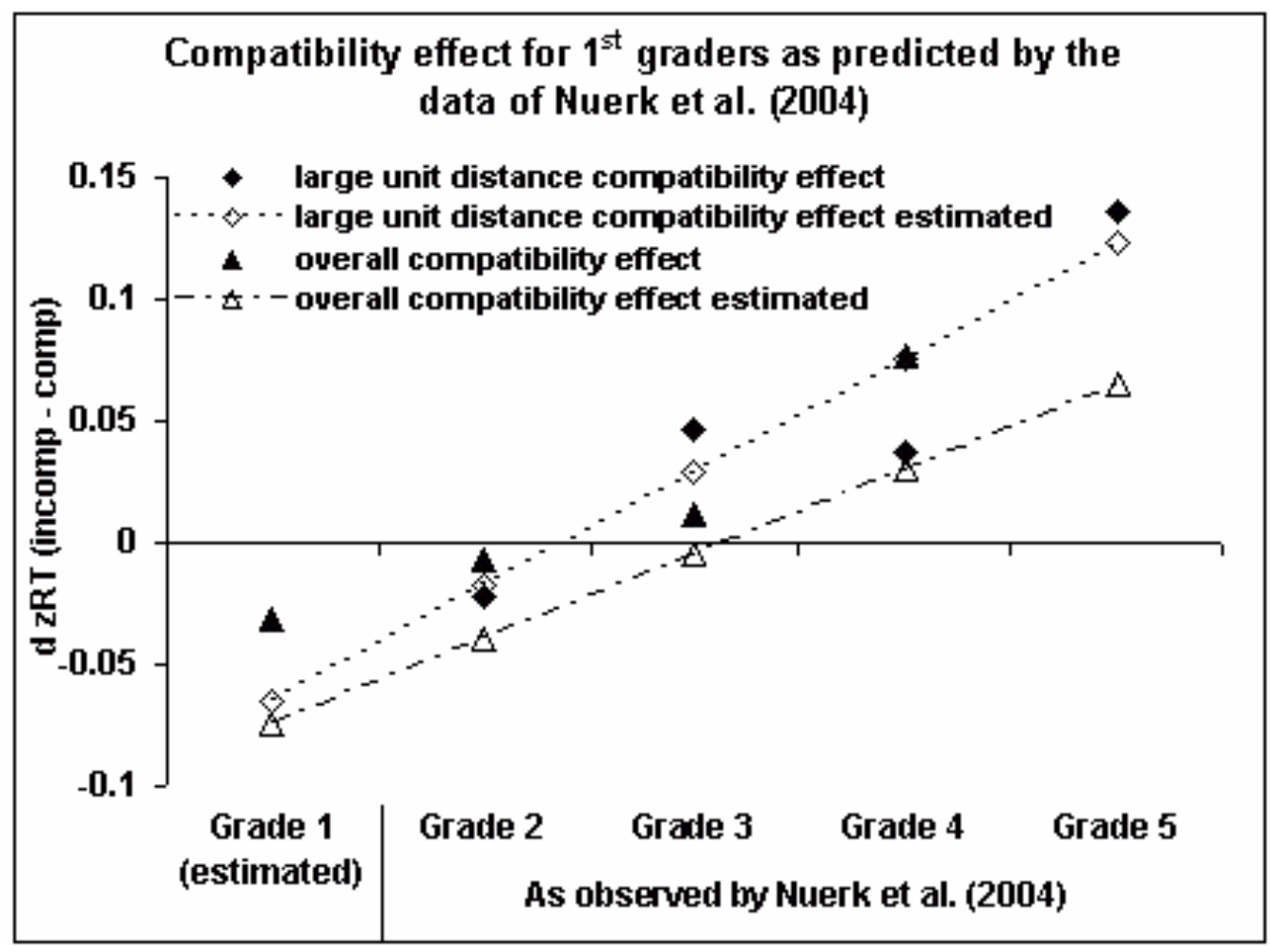

Fig. (1). Expected compatibility effect in $1^{\text {st }}$ grade. The compatibility effect in $1^{\text {st }}$ grade was predicted by a linear regression using grade level as predictor and the compatibility effects in grades 2, 3, 4 and 5 as observed by Nuerk et al., 2004 as dependent variable. Indicating a high reliability, the linear regression explains $89 \%(y=-0.10 x+0.02)$ of the variance for the overall compatibility effect and $85 \%(y=-0.11 x+$ 0.05 ) for the compatibility effect in the large unit distance condition which will be used in the current study. 
observed for adults, they could potentially also be important for children. As Nuerk and colleagues [24] did not include within-decade trials in the stimulus set of their experiment, children could have focused their attention on the decadedigits, and thus, suppressed the influence of the irrelevant units. Therefore, younger children may not automatically process two-digit numbers in a sequential left-to-right fashion. Instead, they may use different processing styles in a similar way as adults do. Thus, they may only process the decade digits first when the decade digits are decisive in all trials. On the contrary, one could suggest that very young children just acquiring two-digit numbers are not yet capable of adapting their processing styles to the demands induced by stimulus properties and that their compatibility effects are indices of their numerical development rather than of their strategic adaptation to task demands. These two alternative hypotheses shall be addressed in the current study.

\section{OBJECTIVES}

The objectives of this study were twofold: First, it was intended to extend the results of Nuerk and colleagues [24] obtained for the $2^{\text {nd }}$ to the $5^{\text {th }}$ grade. For this reason we investigated whether already $1^{\text {st }}$ graders process the magnitude of two-digit numbers in a decomposed fashion or holistically at their early stage of two-digit number acquisition. Second, the current study pursued the question whether a sequential processing style as reported by Nuerk and colleagues [24] for $2^{\text {nd }}$ graders is an adapted processing style (i.e., depending on the stimulus set employed) or is universally applied (i.e., independent of the stimulus set and only depending on the stage of numerical development). To address this second question, the proportion of within-decade trials (e.g., 43_48) was changed as compared to the design of Nuerk et al. [24]. In the latter study $100 \%$ of the number pairs were betweendecade pairs (e.g., 34_48) so that the decade digit was decisive in all trials and the unit digit was irrelevant in all trials. To avoid an attentional bias towards the decade digits, within-decade trials (e.g., 43_49) were added in the current study.

On the basis of this stimulus set change, evaluating the distinction between strategic (stimulus-driven) adaptation of processing styles and their universal developmental is quite simple: If different sets of stimuli lead to differing results, sequential processing is a processing style adapted to the properties of the used stimuli set and does not reflect a processing style prevalent at a given age. If the different sets of stimuli lead to the same results (i.e., a negative compatibility effect in younger children), two-digit number processing in young children is not flexible and adaptive as in adults. Instead, it follows a fixed and invariant processing style which is always applied, no matter, whether this processing style is beneficial in the experimental context or not.

\section{METHODS}

The reported experiment was conducted as part of a larger study investigating the early development of numeracy. Children were tested in different tasks such as transcoding, two-digit number comparison and a number line task. Additionally, verbal (letter repetition task) and visual-spatial (Corsi block tapping task) working memory as well as math anxiety was assessed. This article will focus on the number comparison task.

\section{Participants}

130 (67 boys and 63 girls) Austrian first graders participated in the current study. All Children were of Austrian nationality (i.e., speaking German as their native language) and had normal or corrected to normal vision. Mean age was 7 years and 4 months $(\mathrm{SD}=7.1$ months; range $[6 ; 5-8 ; 7]$ years).

Children with an IQ [as assessed by the CFT1; 25] more than 1 SD below the average were excluded from the initial sample. This affected 2 children.

\section{Stimuli and Design}

The stimulus set consisted of 80 two-digit number pairs between 21 and 98. Decade distance (small: 1 - 3 vs. large: 4 - 8, e.g., 32_57 vs. 32_87) and unit-decade compatibility (compatible, e.g., 32_57 vs. incompatible, e.g., 37_62) were manipulated in a $2 \times 2$ within participant design. Unit distance was not varied in the current study; i.e., only number pairs with large unit distances (i.e., 4 - 8) were used. Additionally, a set of 40 within-decade pairs (e.g., 45_48) was included. In these trials participants need to process the unit digits to come to the correct decision. So, in contrast to the study of Nuerk and colleagues [24], in which decade digits were decisive in all trials, in the current study children had to pay attention to both, decade and unit digits. The 120 items were presented in random order. Neither multiples of ten (e.g., 50) nor tie numbers (e.g., 33) were included in the stimulus set. Moreover, each number pair consisted of four distinct digits, except for the within-decade pairs with identical decade digits. Overall distance and problem size were matched between the respective stimulus groups.

\section{Procedure}

The experiment was carried out during school hours in one-on-one sessions in a separate room. The experiment was run on a notebook with children sitting approximately $50 \mathrm{~cm}$ from the screen. All numbers were shown in Arabic notation using Arial font (size 60). The numbers were positioned above each other separated by $6.3 \mathrm{~cm}$. Successive trials were separated by an ISI of $560 \mathrm{~ms}$. In the magnitude comparison task children were to determine the larger of two two-digit numbers by pressing a corresponding response key. In congruency with the presentation format on the screen the " $Z$ " key of a standard keyboard had to be pressed when the top number was the larger one whereas a larger bottom number was to be indicated by pressing the "N" key. Instructions focused on both speed and accuracy. The critical trials were preceded by ten practice trials.

\section{Analysis}

Response latencies and error rates were analyzed in a $2 \times 2$ repeated measurements ANOVA involving the factors compatibility status (compatible vs. incompatible) and decade distance (small vs. large).

For all analyses only reaction times (RT) followed by a correct response were used (average error rate: $20.64 \%$ ). A 


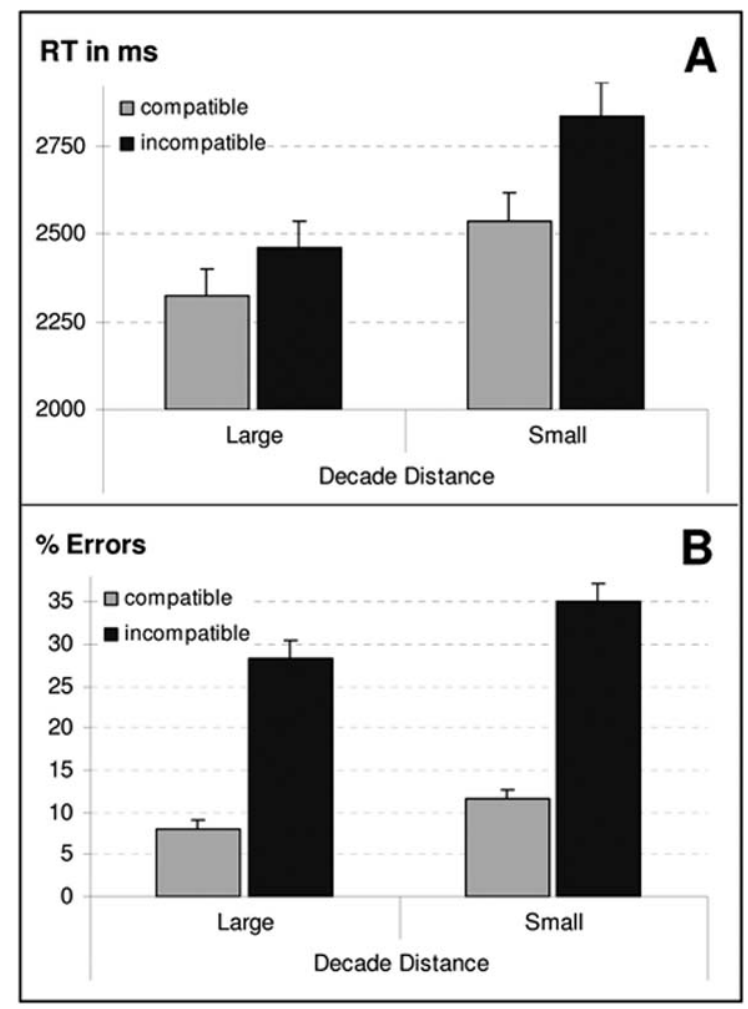

Fig. (2). Reliable effects of decade distance and unit-decade compatibility can be observed for response latencies (Panel A) and error rates (Panel B). Error bars reflect 1 standard error of the mean (SEM).

subsequent trimming procedure eliminated trials with RTs smaller than $200 \mathrm{~ms}$ and longer than $6000 \mathrm{~ms}$. In a second step, trials with an RT exceeding 3 standard deviations above or below a participant's individual mean were excluded from further analysis. This trimming procedure affected $1.01 \%$ of all responses. When a child's performance was below chance level in one condition (> $50 \%$ errors), the mean RT of this condition was excluded from the analysis. The resulting missing values were estimated using a regression model based on the remaining 3 conditions. 5 children $(3.90 \%)$ were excluded from the RT analysis as they performed below chance level in more than one condition.

Means and standard deviations of latencies were found to vary considerably between participants. As such large variability could inconsistently drive effects in one or the other direction, a z-transformation on individual item RTs using the individual participants' mean and standard deviation for standardization was conducted to approximate normal distribution (see Nuerk et al. [24], for the same procedure). Error probability was arcsine transformed prior to the analyses to approximate normal distribution.

\section{RESULTS}

Number comparison was reliably influenced by the factors compatibility status and decade distance in all analyses. The ANOVA revealed a strong effect of decade distance for both speed and accuracy [see Fig. $(2)$, RT: $F(1,122)=120.68$, $p<.001$, Panel A; errors: $F(1,127)=46.17, p<.001$, Panel B]: Number pairs with a large decade distance were responded to faster (294 ms) and more accurately (5\% fewer errors) than number pairs with a small decade distance. Additionally, the compatibility effect was highly significant for RT and error rates [RT: $F(1,122)=28.04, p<.001$; errors: $F(1,127)=124.39, p<.001]$. This indicated that the first graders assessed in the present study responded to faster $(217 \mathrm{~ms})$ and more accurately ( $22 \%$ fewer errors) to compatible as compared to incompatible number pairs. The interaction of decade distance and compatibility did not reach significance $[\text { RT: } F(1,122)=1.98, p=.16 \text {; errors: } F(1,127)<1]^{1}$.

To summarize, strong compatibility effects for latencies and error rates were observed. Because overall (holistic) distance was matched between compatible and incompatible trials, the holistic account cannot explain the compatibility effect observed for first graders here. Therefore, we suggest that already first graders process numbers in a decomposed fashion and not only as one holistic entity. With regard to the second aim of the study, the results seem to suggest that the stimulus properties seem to lead to strategic adaptations of processing styles already in first graders. While Nuerk and colleagues [24] have observed a negative compatibility effect for young children (indicating sequential processing), we observed a positive compatibility effect here (indicating parallel processing). Thus, the results indicate that two-digit number processing may be stimulus-driven and strategically adaptive.

\footnotetext{
${ }^{1}$ As error rates were considerably high the analyses were rerun incorporating only those children that committed $10 \%$ errors or less. An identical pattern of results was observed in these additional analyses: standard decade distance effect [RT: $F(1,51)=$ $71.64, p<.001$; errors: $F(1,51)=18.90, p<.001]$; standard compatibility effect [RT: $F(1,51=7.71, p<.01$; errors: $F(1,51)=46.90, p<.001]$; interaction of decade distance and compatibility [RT: $F(1,51)<1$; Errors: $F(1,51)=1.06, p=.31$ ].
} 


\section{A First Step Exploring Individual Processing Styles in Two-Digit Number Comparison}

As for the above mentioned analysis RT was averaged over all participants, individual differences in processing style were treated as random error. Therefore, an individual analysis differentiating sequential and parallel processing was conducted incorporating only those children with no missing data. Compatibility effects were computed separately for large and small decade distances ${ }^{2}$. Thereby, three groups could be distinguished: (i) 28 children consistently exhibited reliable standard (positive) compatibility effects in both decade distance conditions indicating parallel processing of tens and units throughout the comparison task. (ii) 12 showed a negative compatibility effect with incompatible trials being responded to faster than compatible trials consistently in both decade distance conditions reflecting sequential processing of tens and units. (iii) 30 children exhibited differing compatibility effects (i.e., positive vs. negative) depending on decade distance condition: When decade distance was large, sequential processing as indicated by a negative compatibility effect was more prominent whereas for small decade distances parallel processing as reflected by a positive compatibility effect was favored. However, for some children this was vice versa. Such a pattern may indicate a mixed or inconsistent processing style.

Such a subdivision into three processing style groups could indicate true processing differences, but it could also be just a capitalization on measurement error. However, if the above subdivision into processing style groups is just due to random measurement error, the error distribution of these groups with regard to the compatibility effect should also be random. This means, these groups should not differ systematically in respect of the compatibility effect for errors. The subsequent analysis tested this assumption.

A repeated measures ANOVA was run on arcsine transformed error rates incorporating the within subject factors decade distance (small vs. large) and compatibility status as well as the between subject factor processing style group (parallel, sequential or mixed; see [26] for the case of differing $\mathrm{n}$ in repeated measures ANOVA designs). In addition to strong standard effects of compatibility $[8.4 \% ; F(1,67)=$ $50.30, p<.001]$ and decade distance $[4.8 \% ; F(1,67)=$ $22.04, p<.001]$, a reliable interaction between compatibility status and processing style group was observed $[F(2,67)=$ 5.90; $p<.01$, see Fig. (3)]. Bonferroni-Holm [27] corrected $t$-tests showed that the compatibility effect in the parallel processing group was reliably larger than that for the sequential or mixed processing group $\left[t_{\text {parallel vs. sequential }}(38)=2.60\right.$, $\left.p<.05 ; t_{\text {parallel vs. mixed }}(38)=3.31, p<.001\right]$, whereas the latter two groups did not differ in terms of their compatibility effect $\left[t_{\text {sequential vs. mixed }}(38)=0.42, p=.67\right]$.

\section{DISCUSSION}

The results of the current study are informative in two aspects: Concerning the first objective whether children process two-digit numbers holistically or in decomposed manner at early stages of number acquisition, the findings

${ }^{2}$ Note that these compatibility effects are based on independent, non-overlapping stimulus sets. are not consistent with the assumption of holistic two-digit number magnitude processing in children. Clear evidence for separate processing of tens and units was observed for $1^{\text {st }}$ graders, which further extends the findings of Nuerk and colleagues [24]. The analyses of response latencies and accuracy showed reliable compatibility effects: Compatible number pairs, in which comparing the decade digits magnitudes biased the decision in the similar direction as the comparison of the unit digits magnitudes were responded to faster and more accurately than incompatible trials. When children processed the two to-be-compared numbers holistically, no differences between compatible and incompatible trials should have emerged, as overall distance was held constant between the respective stimulus groups. These results suggest that $1^{\text {st }}$ graders compared the magnitudes of tens and units separately rather than holistically.

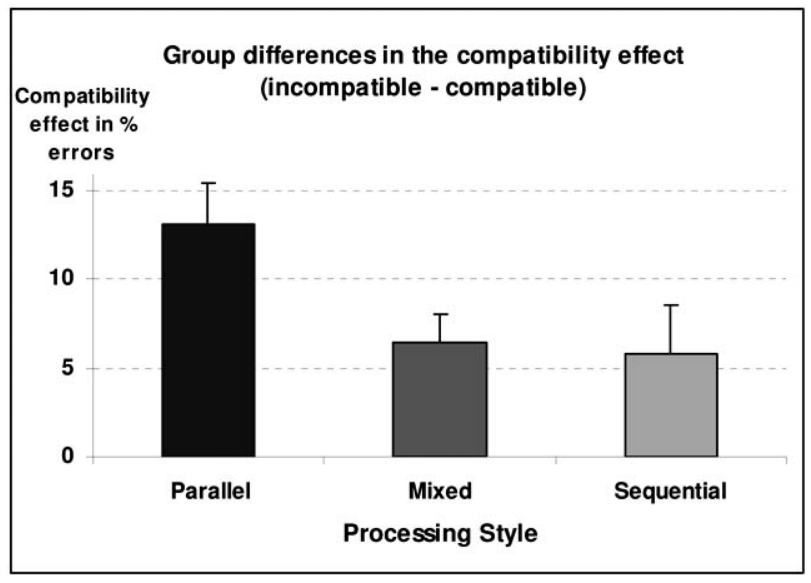

Fig. (3). The unit-decade compatibility effect as observed for error rates separated for the three processing style groups.

Regarding the second objective of distinguishing whether children employed a sequential (left-to-right) or parallel processing style in comparing tens and units, the direction of the observed compatibility effect is informative. When sequential processing of tens and units took place a negative (reversed) compatibility effect (i.e., incompatible trials are processed faster than compatible trials) would have been expected. On the other hand, when children processed tens and units in parallel, a positive (standard) compatibility effect should have been observed (see introduction for a detailed description). Based on the results of Nuerk et al. [24] one would expect decomposed sequential processing of tens and units for two reasons: (i) as the authors observed a negative compatibility effect for second graders they conclude that by this age sequential processing of two-digit numbers is prevalent. (ii) As Fig. (1) depicts [see also Fig. (3) (p. 1207) in 24] there is a linear trend in the size of the compatibility effect for RT indicating that the compatibility effect is continuously increasing with age: It starts with a negative effect for second graders, changes to a positive effect for third graders and ends as an even more pronounced positive compatibility effect in fifth graders. Following this, an increased negative compatibility effect for first graders compared to second graders was expected indicating sequen- 
tial left-to-right processing of the single digits magnitudes of tens and units in younger children. However, the current results did not follow the trend observed by Nuerk et al. [24]. Instead, we found a regular compatibility effect meaning that compatible number pairs were responded to faster and less error prone than incompatible pairs. This suggests tens and units to be processed in a decomposed fashion and in parallel, rather than sequentially. Additionally, these results corroborate the assumption of an adaptive use of processing styles. An additional set of within-decade filler items was used in the current study. As outlined in the introduction this might have prevented children from exclusively focusing on the decade digits, thus leading to a more pronounced processing of the unit digits which may have driven the regular compatibility effect. Taken together, one could conclude that the processing styles children use to determine the larger of two two-digit numbers are adaptive to the stimuli set chosen.

\section{Reasons for Different Compatibility Effects in Different Studies}

It is important to note that the idea that even very young children adjust their processing style strategically in response to task demands is not a generally new idea. Siegler [28] suggested in his overlapping waves model that different strategies are available within the same developmental phase. This means that children at a given point in their development are able to use different strategies and adapt their strategy depending on the task at hand. The results of the current study seem to support these assumptions of Siegler [28].

The two different strategies for processing two-digit numbers relevant for Siegler's model [28] in our study are the sequential-decomposed and the parallel-decomposed processing style. The sequential processing style implies that the tens are processed first and predominantly while the parallel processing style indicates that tens and units are processed simultaneously and in parallel. As outlined in the introduction, a sequential processing style is indexed by a negative compatibility effect while a parallel processing style is indexed by a regular positive compatibility effect.

What would be the most appropriate processing style for different stimulus demands. When no within-decade trials were included in the stimuli set [as in Nuerk and colleagues, $24]$, the decade digits are $100 \%$ relevant and the unit digits are $100 \%$ irrelevant. Consequently, processing the decade digit first and predominantly (sequential processing style) is the most appropriate processing style for the stimulus set used by Nuerk and colleagues [24]. However, in the current study within-decade trials (e.g., 43_48) were added to the stimulus set. Thus, decade digits were no longer $100 \%$ relevant (for 43_48, the magnitude comparison cannot be solved by comparing the decade digits only), but unit digits were also relevant. Thereby, processing both decade and unit digits (in parallel) is the most appropriate processing style for the stimulus set of the current study, because both digits are at times relevant to come to the correct solution. The integration of the data of Nuerk et al. [24] and that of the current study suggests that even very young children process two-digit numbers sequentially when most appropriate, but are also able to process two-digit numbers in parallel when appropriate.

This means that even very young children (i.e., first graders) are able to use different processing styles and to strategically adapt their processing style depending on task requirements. In this sense, the current study corroborates Siegler's [28] assumption of adaptive strategy choices: Even in a task as simple as number comparison children seem to strategically adapt their processing style.

\section{Possible Implications of Decomposed Two-Digit Number Processing on Numerical Development}

Basically, decomposed processing of two-digit numbers reflects that tens and units are processed separately. However, to fully encode a given number, it is not sufficient to process the constituent digits separately. Rather, the single digits' magnitudes of tens and units do not only have to be identified but also they have to be integrated correctly (i.e., at their respective position) into the framework of the placevalue structure of the Arabic number system. For instance, when processing the number 37 the magnitudes of the two digits 3 and 7 have to be encoded and they have to be put together in the correct order to represent 37 and not 73. Following this argument the unit-decade compatibility effect serves as an index for the ability to correctly integrate the magnitudes of tens and units into the place-value structure of the Arabic number system: the larger the compatibility effect, the more demanding is this integration process as it takes longer to resolve the incongruent decision biases elicited by the separate comparison of tens and units. Yet, such integration processes are not only necessary in number comparison. Even in a task as basic as transcoding a number from one notation to another (e.g. writing down a number in symbolic digits to dictation) requires successful integration of tens and units to transcode the lexical components of the heard number word in its corresponding digits. Such successful integration of the single digits into the placevalue structure is even more demanding in number word systems in which the order of tens and units is reversed in spoken number words as compared to the digital notation (e.g., German, Dutch, Arabic, etc.). In this vein, Zuber, Pixner, Moeller, and Nuerk [29] found that about one half of all the transcoding errors committed by German-speaking first graders were inversion-related. However, correct application of place value information is also required in the basic arithmetic operations. Consider the case of addition (see e.g., Domahs et al. [30, 31] for the use of place-value information in multiplication). In two-digit addition problems a carry is required whenever the sum of the unit digits exceeds 9 (e.g., $25+58=83$ vs. $31+52=83$ ). The carry operation is executed by adding 1 (representing the decade digit of the unit sum) to the sum of the decade digits of the summands. For the above example $25+58$ the sum of the unit digits $(5+8)$ is 13 , so the unit digit of the result is 3 and the decade digit of the result is derived by updating the sum of the decade digits of the summands by 1 (i.e., the decade digit 
of the unit sum: $2+5+1=8$ ). This means that in carry addition problems not only successful identification and integration of the single digits of the summands into the place-value structure of the Arabic number system is required. Additionally, updating the sum of the decade digits of the summands by the carried decade digit of the unit sum is necessary, which nicely illustrates the importance of place-value understanding in basic arithmetical operations. Similar place-value integrations are necessary in subtraction (borrowing effect), multi-digit division procedures or the multi-digit number bisection task [32].

Taken together, we suggest that the compatibility effect observed in first grade (as well as the number of inversionrelated transcoding errors) may serve as a predictor of later arithmetic performance. In particular, as the compatibility effect reflects mastery of the place-value structure of the Arabic number system, it should be an especially valid predictor of performance in tasks that do require successful understanding and application of place-value information such as carry addition problems. Yet, this proposition still needs to be evaluated by future research investigating the importance of place-value understanding on children's numerical development.

\section{A Final Comment on Potential Inter-Individual Differences}

The above paragraph is only referring to the compatibility effects observed on average at a given age. However, even within the same age cohort different individuals may use different processing styles which might be intraindividually stable. To make a first step towards examining inter-individual differences, we categorized three processing style sub-groups of children on the basis of their RT data: a parallel processing group, a sequential processing group, and a mixed processing group. It was observed that the parallel processing group (as indexed by RT) also exhibited a reliably larger compatibility effect for error rates compared to that observed for the other two groups. This seems to suggest that the parallel, sequential or mixed processing styles used by the different groups may not be due to random RT measurement error, but are indexes of intra-individually stable processing styles which can be reliably observed for different dependent variables. Clearly, in the future, one should examine whether individually stable processing styles cannot only be shown for different dependent variables within one task, but also for different (independent) tasks. Nevertheless, these results seem to give a first indication that even basic numerical cognition such as number magnitude comparison may be influenced by inter-individually different processing styles.

\section{CONCLUSIONS AND PERSPECTIVES}

Children were found to be able to process tens and units separately already in $1^{\text {st }}$ grade. The current data indicate that previous findings suggesting sequential processing of tens and units in children seemed to be induced by a particular stimulus set rather than reflecting universal processing characteristics. Even young children (first graders) seem to be able to adapt their individual processing styles depending on stimulus properties. Consequently, in future experiments adaptive effects induced by the used stimulus set should be considered.

Finally, potential inter-individual differences indicated that not even in a task as simple as magnitude comparison, all children employed the same processing style at a given point of development. In our view, these analyses suggest that inter-individual differences may deserve greater attention in future examinations (e.g., about the stability of such differences across different, independent tasks). Interindividual differences between children of the same age may not be just random measurement errors as which they are treated in many standard statistical examinations.

\section{ACKNOWLEDGEMENTS}

This research was supported by a grant from the AKTION Österreich-Tschechien (45p13) to Silvia Pixner und Hans-Christoph Nuerk. We wish to thank two anonymous reviewers for helpful suggestions and comments on an earlier version of the manuscript.

\section{REFERENCES}

[1] Siegler RS, Robinson, M. The development of numerical understandings. Adv Child Dev Behav 1982; 16: 241-312.

[2] Rubinsten O, Henik A, Berger A, Shahar-Shalev S. The development of internal representations of magnitude and their association with Arabic numerals. Exp Child Psychol 2002; 81: 74-92.

[3] Kaufmann L, Nuerk HC. Interference effects in a numerical Stroop paradigm in 9- to 12-year-old children with ADHD-C. Child Neuropsychol 2006; 12: 223-43.

[4] Kaufmann L, Koppelstaetter F, Siedentopf C, et al. Neural correlates of the number-size interference task in children. Neuroreport 2006; 17: 587-91.

[5] Dehaene S, Bossini S, Gireaux P. The mental representation of parity and numerical magnitude. J Exp Psychol Gen 1193; 122: 371-96.

[6] V Galen MS, Reitsma P. Developing access to number magnitude: A study of the SNARC effect in 7- to 9-year-olds. J Exp Child Psychol 2008; 101: 99-113.

[7] Berch DB, Foley EJ, Hill RJ, Mcdonough-Ryan PM. Extracting parity and magnitude from Arabic numerals: developmental change in number processing and mental representation. J Exp Child Psychol 1999; 74: 286-308.

[8] Ganor-stern D, Pinhas M, Tzelgov J. Comparing two-digit numbers: The importance of being presented together. Q J Exp Psychol 2008; 62: 444-52.

[9] Moeller K, Nuerk HC, Willmes K. Internal magnitude representation is not holistic, either. Eur J Cogn Psychol DOI: 10.1080/ 09541440802311899.

[10] Nuerk HC, Weger U, Willmes K. Decade breaks in the mental number line? Putting the tens and units back in different bins. Cognition 2001; 82: B25-33.

[11] Zhang J, Wang H. The effect of external representation on numeric tasks. Q J Exp Psychol 2005; 58: 817-38.

[12] Nuerk HC, Willmes K. On the magnitude representation of twodigit numbers. Psychol Sci 2005; 47: 52-72.

[13] Hinrichs JM, Yurko DS, Hu JM. Two-digit comparison: use of place information. J Exp Psychol Hum Percept Perform 1981; 7: 890-901.

[14] Moeller K, Fischer, MH, Nuerk HC, Willmes K. Sequential or parallel decomposed processing of two-digit numbers? Evidence from eye-tracking. Q J Exp Psychol 2009: 62: 323-34.

[15] Dehaene S, Dupoux E, Mehler J. Is numerical comparison digital? Analogical and symbolic effects in two-digit number comparison. J Exp Psychol Hum Percept Perform 1990; 16: 626-41.

[16] Moyer RS, Landauer TK. Time required for judgments of numerical inequality. Nature 1967; 215: 1519-20. 
[17] Nuerk HC, Weger U, Willmes K. On the perceptual generality of the unit-decade compatibility effect. Exp Psychol 2004; 51: 1-8.

[18] Nuerk HC, Weger U, Willmes K. Language effects in magnitude comparison: Small, but not irrelevant. Brain Lang 2005; 92: 26277.

[19] Ratinckx E, Nuerk, HC, Van Dijck JP, Willmes K. Effects of the interhemispheric interaction in two-digit number processing. Cortex 2006; 42: 1128-37.

[20] Verguts T, De Moor W. Two-digit comparison: decomposed, holistic, or hybrid? Exp Psychol 2005; 52: 195-200.

[21] Wood G, Nuerk HC, Willmes K. Neural representation of two digit numbers: a parametric fMRI study. NeuroImage 2006; 29: 358-67.

[22] Knops A. On the structure and neural correlates of the numerical magnitude representation and its influence in the assessment of verbal working memory. Ph.D. diss., Technical University Aachen 2006.

[23] Nuerk HC, Weger U, Willmes K. A unit-decade compatibility effect in German number words. Curr Psychol Lett Behav Brain Cogn 2002; 2: 19-38.

[24] Nuerk HC, Kaufmann L, Zoppoth S, Willmes K. On the development of the mental number line: More, less, or never holistic with increasing age? Dev Psychol 2004; 40: 199-211.
[25] Catell RB, weiss RH, Osterland J. Culture Fair Test 1. Göttingen: Hogrefe 1997.

[26] Mycroft RH, Mitchell DC, Kay J. An evaluation of statistical procedures for comparing an individual's performance with that of a group of controls. Cogn Neuropsychol 2002; 19: 291-9.

[27] Holm S. A simple sequentially rejective multiple test procedure. Scand J Statist 1997; 6: 65-70.

[28] Siegler RS. Emerging minds: the process of change in children's thinking. New York: Oxford University Press 1996.

[29] Zuber J, Pixner S, Moeller K, Nuerk HC. On the languagespecificity of basic number processing: Transcoding in a language with inversion and its relation to working memory capacity. J Exp Child Psychol 2009; 102: 60-77.

[30] Domahs F, Delazer M, Nuerk HC. What makes multiplication facts difficult. Problem size or neighbourhood consistency? Exp Psychol 2006; 53: 275-82.

[31] Domahs F, Domahs U, Schlesewsky M, et al. Neighborhood consistency in mental arithmetic: behavioral and ERD evidence. Behav Brain Funct 2007; 3: 66.

[32] Nuerk HC, Geppert BE, Herten VM, Willmes K. On the impact of different number representations in the number bisection task. Cortex 2002; 38: 691-715.

(C) Pixner et al.; Licensee Bentham Open.

This is an open access article licensed under the terms of the Creative Commons Attribution Non-Commercial License (http://creativecommons.org/licenses/by-nc/3.0/) which permits unrestricted, non-commercial use, distribution and reproduction in any medium, provided the work is properly cited. 